\title{
Short and long term behavior of functionally filled polymeric insulating materials for HVDC insulators in compact gas-insulated systems
}

\author{
Michael Tenzer, Maximilian Secklehner, Volker Hinrichsen \\ TU Darmstadt, High Voltage Laboratories, \\ Landgraf-Georg-Str.4, D-64283 Darmstadt, Germany.
}

\begin{abstract}
Conventional insulators optimized for high-voltage alternating current transmission tend to accumulate surface and volume charges in direct voltage applications. This is especially true for gas-insulated systems, where the surrounding gas is extremely dry, thus having very low conductivity. This may result in a strong decrease of the dielectric strength of the insulators and can lead to dielectric breakdown, especially when polarity reversals are applied. Main challenges for the development of HVDC insulators are avoiding surface and volume charge accumulations and featuring both suitable capacitive and resistive field distributions. The use of polymeric insulation materials filled with functional fillers of defined low and possibly non-linear, field dependent electric conductivity avoids these charge accumulations.

Several specimens of polymeric insulation materials of different, controlled conductivities for high field stress applications were produced and experimentally investigated for this contribution. Since electric conductivity depends on parameters such as temperature, humidity or long term ageing, the longterm behavior of the specimens was investigated in $1000 \mathrm{~h}$ tests under temperature and electrical field stress. Furthermore, tests in a high-voltage gas insulated test setup were performed in order to determine the dielectric strength of the filled polymers under high electric stationary and transient fields as present in gas insulated systems. Results of these investigations are presented and discussed in detail in this contribution.
\end{abstract}

\section{Introduction}

Todays' available insulating materials, especially epoxy resin, have been optimized for field stress under alternating voltages. When designing a material for direct voltage applications, a higher conductivity $\left(\kappa \approx 10^{-11} \ldots 10^{-13} \mathrm{~S} / \mathrm{m}\right)$ is needed in order to achieve both a fast decay of surface charges and a better field distribution. One way of achieving a higher, but welldefined conductivity of polymeric insulation materials is the application of functional fillers in the polymeric matrix. A possible and still new approach is the use of microvaristors for this purpose. Microvaristors are small, sintered particles of doped $\mathrm{ZnO}$ having a welldefined, intrinsic non-linear conductivity $\kappa(E)[1]$. The non-linearity of microvaristors is expressed by the exponent of non-linearity, $\alpha$, which ranges up to $\alpha \approx 30$ [1]. They become increasingly conductive above a certain level of the applied electric field, the "switching field strength". One main advantage of microvaristors used as functional fillers is the fact that the conductivity is mainly determined by the internal potential barriers within the particles, rather than by effects at the boundaries between the particles, and their electrical characteristic is tuned by variation of the doping elements or the sintering parameters [1].

For d.c. applications, the filling degree must be chosen above the percolation threshold to provide conduction paths inside the polymer. Microvaristors are to date typically applied in silicone rubber for field grading applications, i.e. stress cones for cable terminations [2], [3], [4].

For high-field applications, such as in compact gasinsulated systems, microvaristors of very high switching field strength are necessary. Furthermore, for application in epoxy resin, the particle size must be as small as possible in order to avoid any sedimentation during the manufacturing process and thus to achieve a perfectly homogeneous distribution of the electrical parameters. For this reason, a batch of special microvaristors was manufactured and delivered by one manufacturer. Typical for these particles are a lower nonlinearity exponent $\alpha$ and a lower particle diameter. This type of microvaristors, named "Type A" here, is mainly studied for this contribution.

\section{Manufacturing of Microvaristor-filled Specimens}

For the experimental investigations, microvaristor-filled epoxy resin specimens with "Type A" particles are manufactured. In contrast to so far "Standard" microvaristors, the "Type A" particles have very small diameters $(d<40 \mu \mathrm{m})$ and consist of a mixture of small spherical particles $\left(d_{50} \approx 20 \mu \mathrm{m}\right)$ and a large amount of very small fragments $\left(d_{50} \approx 3 \mu \mathrm{m}\right)$.

A bisphenol A type epoxy resin is used for the polymeric matrix. The epoxy resin, mixed with hardener and microvaristors "Type A", is vacuum-cast into a cylindrical mold of $45 \mathrm{~mm}$ in diameter and a height of $200 \mathrm{~mm}$. As aforementioned, the filling degree of the microvaristors is chosen well above the percolation threshold in order to form conductive particle-particle paths within the polymeric specimens. The homogeneity of each specimen is verified by measuring the $E-J$ 
characteristic of thin slices from top and bottom of the cylindrical specimens.

\section{Experimental Results}

\subsection{Measurement of the $E-J$ Characteristics}

Thin slices of $2 \mathrm{~mm}$ height are cut out from the manufactured cylindrical specimens. By using silver conductive painting, guarded electrodes for bulk

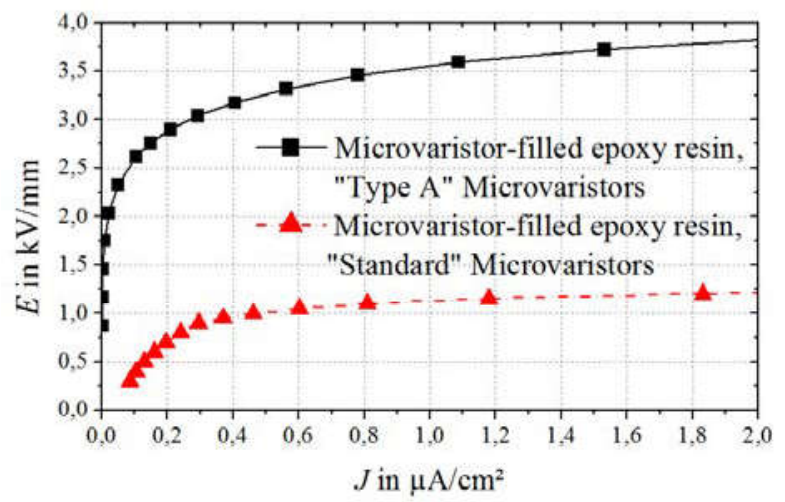

Figure 1: Characteristic d.c. $E-J$ curve of microvaristorfilled epoxy resin insulators with "Standard" microvaristors and of the microvaristor-filled epoxy resin filled with "Type A" microvaristors.

conductivity measurements according to IEC 60093 [5] are applied to the slices, and the d.c. $E-J$ curves are then recorded and are plotted in the graph of Figure 1.

Comparing the $E-J$ curve of the "Type A"microvaristor-filled epoxy resin (EPR) specimen with the curve of the "Standard" microvaristor-filled EPR specimen, it can be seen that conductivity $\kappa(E)$ and nonlinearity exponent $\alpha$ are reduced in case of the "Type A" microvaristors.

It can thus be stated that the manufacturer has succeeded in producing microvaristors of much higher switching field strength $E_{\mathrm{b}}$ (defined as the field strength at which a current density $J_{\mathrm{DC}}=1 \mu \mathrm{A} / \mathrm{cm}^{2}$ is reached), resulting in a value of $E_{\mathrm{b}} \approx 3.5 \mathrm{kV} / \mathrm{mm}$ of the overall insulator. However, it is obvious that for future applications in gas-insulated systems $E_{\mathrm{b}}$ must reach even higher values, since it has to be significantly higher than the maximal operating field strength of the insulating material in order to avoid damages or electrical breakdowns.

\subsection{High-Field Tests with Direct and Impulse Voltages}

In addition to the measurements of the thin slices presented in Section 3.1, the manufactured microvaristor-filled EPR cylinders are contacted by silver conductive painting on the end faces and then mounted between electrodes in a gas-insulated test setup under $\mathrm{SF}_{6}$ gas. Figure 2, left, shows a photo of such specimen.

Direct voltage tests are carried out on three specimens of this geometry under high field conditions. Up to $E \approx 0.66 \cdot E_{\mathrm{b}}$, the direct currents are stable during the test procedure (several minutes). At a direct field stress of $E=1.25 \cdot E_{\mathrm{b}}$, the specimens are able to withstand the electric stress for more than sixty seconds.

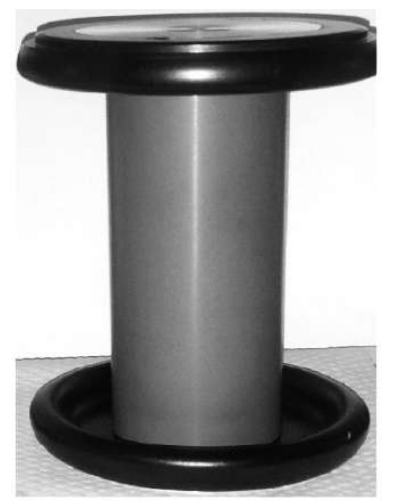

Figure 2: Cylindrical microvaristor-filled EPR specimen with electrodes. Left: before test; right: after SI failure (cracking)

After the direct voltage tests, the specimens are tested by switching impulse voltage $(250 / 2500 \mu \mathrm{s})$, and all specimens pass this test up to peak impulse voltages corresponding to $\hat{E} \approx 2 \cdot E_{\mathrm{b}}$. When a field strength of $\hat{E} \approx 2 \cdot E_{\mathrm{b}}$ is exceeded, the specimens fail by cracking

(Figure 2, right). These results give first ideas how to dimension such insulators with regard to their switching field strength.

\section{Long Term D.C. Investigations}

Two test series of $1000 \mathrm{~h}$ time duration are conducted in order to verify the long-term stability of the "Type A" microvaristor compound. In this case stability is defined as a state where nearly no change of the electrical characteristics, especially the conductivity, takes place. One test is conducted without and the other one with repetitive polarity reversals, while at the same time the specimens are exposed to elevated temperatures.

\subsection{Test Stand for Accelerated Ageing Tests}

The high-voltage source is provided by a software generated sinusoidal signal, which is then amplified and fed to a transformer. Its secondary side high alternating voltage is rectified by a one-way rectifier. To change polarity, the smoothing capacitor is discharged within 17 seconds. Afterwards the circuit is re-energized at opposite polarity.

The specimens are mounted in heating chambers with forced convection. The direct voltage is fed from outside into the chambers by bushings.

The following magnitudes are measured and controlled, respectively: current through and voltage across the 
specimens, temperature and relative humidity inside the chamber.

\subsection{Results of the Unipolar DC Long Term Test}

Five of the slice shaped specimens, as described in Section 2, are tested in each of the three heating chambers. The temperatures are chosen as follows: $\vartheta_{1}=30{ }^{\circ} \mathrm{C}, \vartheta_{2}=60{ }^{\circ} \mathrm{C}, \vartheta_{3}=100{ }^{\circ} \mathrm{C}$. In this first test the electrical contacts of the specimens are implemented as electrodes according to IEC 60093 [5], without additional use of conductive paint. The electrodes are pressed onto the specimens with a force of $F=10.2 \mathrm{~N}$. An electrical field of $E=1.75 \mathrm{kV} / \mathrm{mm}$, corresponding to $0.5 \cdot E_{\mathrm{b}}$, is applied to the specimens.

Figure 3 shows the development with time of current density $J_{\mathrm{DC}}$ of a specimen tested at $\vartheta_{1}=30^{\circ} \mathrm{C}$. It can be seen that current density, and thus electric conductivity, undergo drastic changes. In this case conductivity at the end of the test reaches a value of 27 times the initial value. This behavior is, therefore, extremely unstable.

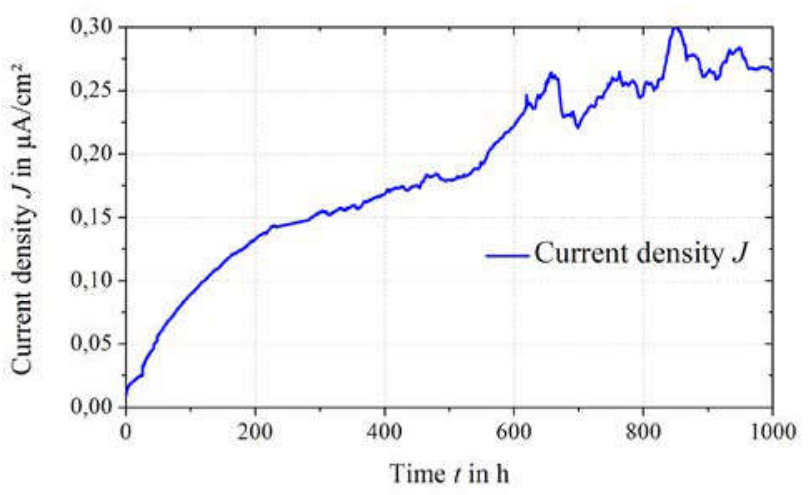

Figure 3: Typical current density over time of one specimen tested at $\vartheta_{1}=30^{\circ} \mathrm{C}$

At higher temperatures current densities also increase with testing time, but with some alternations. While the increase in conductivity is faster in the beginning of the test at higher temperatures, the process slows down after

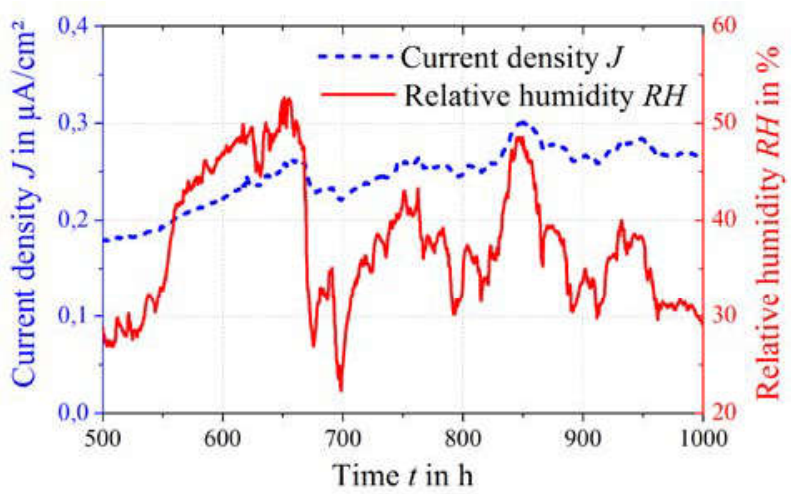

Figure 4: Comparison of current density of one specimen and relative humidity inside the heating chamber over time
$160 \mathrm{~h}$ for the specimens tested at $\vartheta_{2}=60{ }^{\circ} \mathrm{C}$ and after $50 \mathrm{~h}$ for the specimens tested at $\vartheta_{3}=100{ }^{\circ} \mathrm{C}$. However, all specimens show extremely unstable behavior.

After some $600 \mathrm{~h}$, fluctuations in the current density appear, whose origin is further investigated. In Figure 4 the current density of one specimen as well as the relative humidity inside the chamber are plotted over time. These curves are clearly correlated.

Since both test voltage and temperature exhibit only very limited alternations $(\Delta U<2 \% ; \Delta \vartheta<2.8 \%)$, they can be ruled out as possible origins of the current fluctuations. Therefore, it may be assumed that the humidity of the surrounding air has great influence on the conductivity of the microvaristor compound. For epoxy based insulating systems it is also known that power losses increase with humidity [6].

When comparing the specimens' $E$ - $J$-characteristics before and after the test a strong shift becomes evident. The increase of current density is obviously linked to a permanent degradation of the $E$ - $J$-characteristic curve of the compound.

\subsection{Results of the DC Long Term Test with Polarity Reversal}

For the second direct voltage long term test, the following changes are introduced: To achieve better electrical contact the specimens' end surfaces are coated with silver loaded conductive paint such that a guard ring electrode configuration according to IEC 60093 [4] is achieved. Polarity reversals in varying time intervals are introduced as additional test parameter.

Figure 5 shows the typical power loss of one specimen over time. After every polarity reversal power loss decreases, passes through a minimum and exhibits a steady increase afterwards.

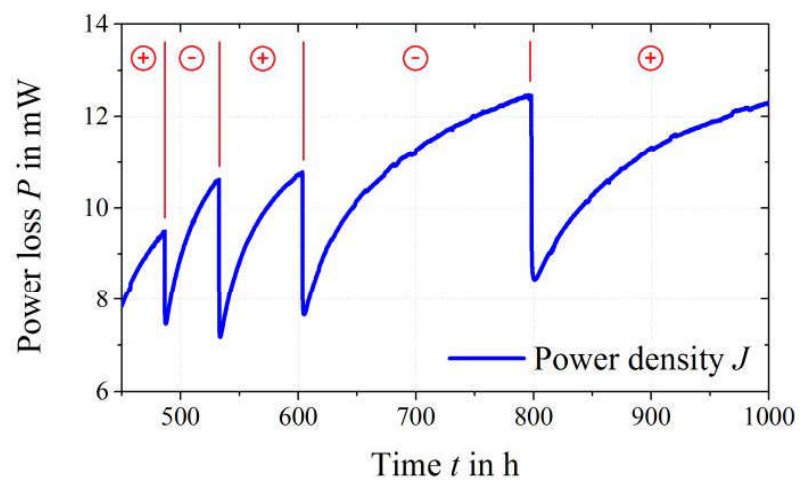

Figure 5: Typical power loss over time of one specimen showing the effect of polarity reversals

This basic behavior is recognized for all specimens, independent of the test temperature. The relative increase of current density over the whole test duration is present but lower than in the comparable tests at unipolar field stress. Polarity reversals thus do not represent a critical stress factor for the tested microvaristor compound, a behavior that is also known for energy MO varistors. 
Relative humidity inside the heating chambers is strictly controlled throughout this second long term test by use of a drying agent. Consequently, no fluctuations of current density can be detected. The coating with conductive paint may have acted as an additional protection against moisture ingress into the compound.

\subsection{Tests of further Microvaristor Compounds}

To understand the reason of the unstable behavior, three further microvaristor compounds are tested at shorter time durations. Both, the applied microvaristors ("Standard", "Type A") and the embedding polymeric matrix $(\mathrm{EPR}, \mathrm{SiR}=$ silicone rubber $)$ are varied. The three additional tests are all carried out at $\vartheta=30{ }^{\circ} \mathrm{C}$ and $E \approx 0.5 \cdot E_{\mathrm{b}}$, respectively.

Figure 6 shows the current densities over time of the four tested compounds normalized to the corresponding initial current density.

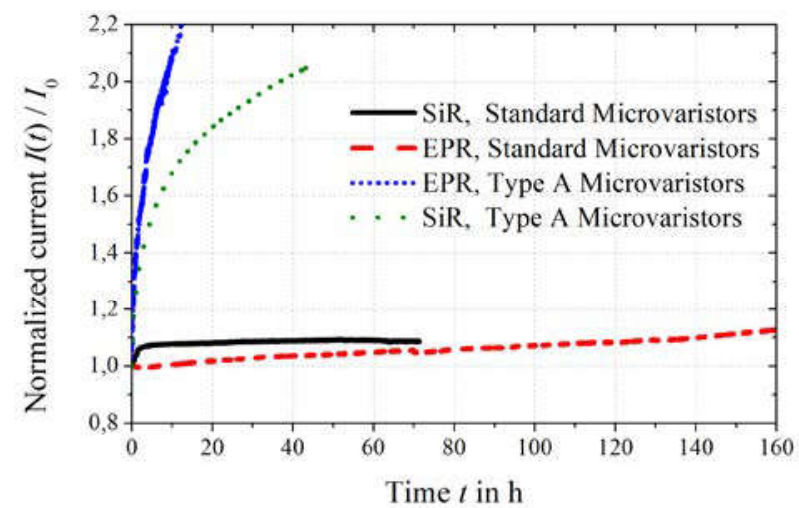

Figure 6: Comparison of the normalized current density over time of the four tested microvaristor compounds

The already discussed EPR insulator filled with "Type A" microvaristors shows the fastest and most extreme increase in current density (broad dotted line). Also, the SiR insulator filled with "Type A" microvaristors behaves unstable (fine dotted line). But both the EPR as well as the SiR compounds containing "Standard" microvaristors show an only moderate or even no increase at all of current density.

It is assumed that the unstable behavior of "Type A" microvaristor-filled compounds is caused by the manufacturing process applied for the "Type A" microvaristors. The high content of fractions, besides the spherical particles, obviously affects the d.c. stability of the resulting compounds. The tests on the specimens filled with "Standard" microvaristors, however, clearly demonstrate that microvaristor-filled compounds can be made stable against direct voltage stress. Appropriate improvements of the manufacturing process seem to be possible and will have to be introduced with the goal to make the high-switchingfield strength microvaristors stable against direct electric field stress.

\section{Conclusions}

It has been shown that it is basically possible to manufacture microvaristor-filled epoxy resin insulators for very high direct electric field stress applications. The experimental results presented in Section 3 have proven their feasibility. However, for high-field insulating materials to be used in compact gas-insulated systems, the $E-J$ characteristic must be further shifted towards higher switching field strengths $E_{\mathrm{b}}$ in order to provide sufficient margins to the expected overvoltage stress in such applications. But it has also been shown that the actual "Type A" microvaristor-filled compounds in their present implementation are not long-time stable under direct voltage stress, whereas the compounds filled with "Standard" microvaristors can obviously be operated in a stable mode under this kind of stress. It is assumed that the d.c. instability is associated with the differing particle structure of the applied "Type A" microvaristors These findings indicate possible ways to solve the problems with d.c. stability. It is obviously not advisable to mechanically treat the sintered microvaristor particles after the sintering process in order to achieve a smaller resulting particle size.

\section{Outlook}

Further investigations on microvaristor-filled insulators are in progress, and the possibilities of manufacturing other types of microvaristors, having high switching field strength $E_{\mathrm{b}}$ and a stable d.c. behavior, are being discussed with the manufacturer. It is assumed that it will be possible to optimize the high-field microvaristors, as the reasons of the instability have been identified.

Furthermore, other types of functional fillers are actually being investigated, which have shown very promising results so far for the intended applications.

\section{References}

[1] L. Donzel, F. Greuter, Th. Christen, "Nonlinear Resistive Electric Field Grading Part 2: Materials and Applications", IEEE Electrical Insulation Magazine, Vol. 27, No. 2, 2011, pp. 18-29.

[2] B. Boettcher, G. Malin, R. Strobl, "Stress control system for composite insulators based on ZnO-Technology", IEEE/PES, Vol. 2, 2001, pp. 776-780

[3] D. Weida, C. Richter, M. Clemens, "Design of $\mathrm{ZnO}$ microvaristor material stress-cone for cable accessories", IEEE Transactions on Dielectrics and Electrical Insulation, Vol. 18, 2011, pp. 1262-1267

[4] U. Amerpohl, M. Kirchner, B. Böttcher, and G. Malin, "Dry type outdoor termination with new stress control management", in session papers CIGRE 2002, Paris, France, paper 21-106, Aug. 2002.

[5] IEC 60093, "Methods of test for volume resistivity and surface resistivity of solid electrical insulating materials" $2^{\text {nd }}$ edition, 1980.

[6] S. Li, L. Liang, J. Li, N. Liu, M.A. Alim, „Characterization of Water absorbed epoxy insulating coating material used in $\mathrm{ZnO}$ varistors by dielectric measurements", Materials Letters, Volume 60, Issue 1, 2006, pp. 114-119. 